ARTICLES

\title{
UN PASTEUR ACTUAIRE? ORDRE DE LA MORTALITÉ, DURÉE DE LA VIE ET RENTES VIAGÈRES DANS L'ORDRE DIVIN DE JOHANN PETER SÜSSMILCH
}

\author{
Jean-Marc RoHRBASSER
}

RÉsumé: L'ordre que le pasteur Süßmilch voit dans les événements démographiques est celui du gouvernement de la Providence, et en particulier l'ordre de la mortalité. Dieu régit la durée de vie des humains et assigne à chacun un juste équilibre entre la crainte de mourir et l' « espoir » de vivre. Ce qui permet à Süßmilch de dégager les notions de vie probable et d'espérance de vie, en vue de traiter leur application principale en arithmétique politique : le calcul des emprunts d'État sous forme de rentes viagères. Il traite ainsi d'une importante question qui relève à la fois de l'histoire du calcul actuariel, du calcul des probabilités, de la démographie statistique, sans oublier les aspects politiques et philosophiques qu'il ne manque pas de souligner : quelle est la nature du contrat qui relie l'individu à l'État? Et surtout, quelle est la nature de celui qui relie la créature à son Dieu? Cette étude des régulạrités contingentes mais optimales que la Providence instaure dans le monde constitue une importante contribution au courant physico-théologique.

Mots-CLÉs : ordre, démographie, statistique, rentes viagères, physico-théologie.

ABSTRACT : The government of Providence builds the order which pastor Süßmilch sees in the demographic events and especially the order of mortality. God governs the length of human life and assigns to each person a just balance between fear of death and expectation of life. This authorizes Süßmilch to clarify the notions of probable life and life expectancy with the design to treat of their main application in the field of political arithmetic, that is the computations of Government loans under the form of annuities on lives. So he treats of an important question which concerns the history of actuarial calculation as well as the history of probability, of statistics and demography. We cannot forget also the political and philosophical points of view which the pastor underlines vividly: what sort of contract joins the individual together with the State? And, above all, what sort of contract joins the creature and his God? This study of the contingent but optimal regularities which Providence makes in the world builds an important contribution to the physico-theological current.

KEYWORDS : order, demography, statistics, annuities, physico-theology.

Revue de synthèse : $4^{\mathrm{e}} \mathrm{S} . \mathrm{N}^{\circ} 4$, oct.-déc. 1997, p. 385-417. 
Zusammenfassung : Die Ordnung, insbesondere die der Sterblichkeit, die Propst Süßmilch in den demographischen Ereignissen wahrnimmt, wird von der Regierung der Vorsehung gestaltet. Gott selbst regiert die Lebensdauer der Menschen und er bestimmt für jeden einen gerechten Mittelweg zwischen der Furcht vor dem Tod und der Hoffnung auf Leben. Süßmilch erklärt die Begriffe der wahrscheinlichen Lebensdauer und Lebenserwartung, um ihre Anwendung, die in der Rechnung der Staatsanleihen in Gestalt der lebenslänglichen Renten besteht, im Rahmen der politischen Arithmetik zu behandeln. Diese wichtige Frage grenzt an die Geschichte der Versicherungsmathematik, der Wahrscheinlichkeitsrechnung und der Bevölkerungsstatistik. Man darf auch die politischen und philosophischen Gesichtspunkte, die Propst Süßmilch betont, nicht vergessen. Welche Beziehung bindet das Individuum an den Staat? Und welche Beziehung bindet insbesondere die Kreatur an ihren Gott? Diese Studie der zufälligen doch optimalen Regeln, die die Vorsehung in die Welt geführt hat, stellt einen wichtigen Beitrag zur physikotheologischen Strömung dar.

STICHWÖRTER : Ordnung, Demographie, Statistik, lebenslängliche Renten, Physiko-Theologie.

Jean-Marc RoHRBASSER, né en 1948, agrégé de philosophie, est chargé de recherches à l'Institut national d'études démographiques dans l'équipe Histoire de la démographie. Ses recherches portent principalement sur l'œuvre de Johann Peter Süßmilch. Il a traduit et commenté la première édition du Göttliche Ordnung, 1741 (Paris, Institut national d'études démographiques, 1998), et étudié l'histoire des rentes viagères et des tontines et la place de la physico-théologie anglaise et hollandaise dans la pensée allemande, de Leibniz aux Naturphilosophen.

Adresse : INED, 27 rue du Commandeur, 75675 Paris Cedex 14.

Courrier électronique : rohrbass@ined.fr 
Il est une étape relativement méconnue de l'histoire de la statistique démographique et du calcul des probabilités : les spéculations du théologien berlinois Johann Peter Süßmilch (1707-1767) sur la durée de la vie humaine. Elles résultent des réflexions qu'il insère dans son œuvre sur la pratique des emprunts d'État sous forme de rentes viagères ou de rentes viagères en tontines.

Pourquoi Süßmilch? D'abord, parce qu'il est incontestablement, en Allemagne, le père fondateur de la statistique démographique; ensuite, parce que, se situant délibérément dans une perspective historique, il cite et traduit abondamment ses contemporains et prédécesseurs, nous permettant ainsi de comprendre une part du développement des concepts qu'il utilise; enfin, parce que, théologien, il aborde les questions démographiques et statistiques en les replaçant dans le cadre d'une foi qui en constitue les conditions de possibilité.

Pourquoi spécule-t-on sur la durée de la vie humaine? D'abord, parce que les États ont besoin d'argent et que l'emprunt remboursable sous forme de rentes est un moyen simple de rassembler rapidement un important capital disponible pour un usage immédiat; ensuite, parce que chaque individu spécule afin, tout simplement, de s'enrichir; enfin, parce qu'il ne faut pas oublier l'attrait du jeu et du pari, la part d'incertitude que comportent ces pratiques quant au désir, tout humain, d'obtenir quelque lumière sur le futur et d'en espérer quelque illusion de maîtrise.

Sans négliger l'aspect politique, le théologien Süßmilch réfléchit sur la durée de la vie humaine dans un cadre bien précis : celui de la physicothéologie pour qui la nature - pas seulement la nature physique des corps inanimés, voire celle de la «biologie », mais également celle des phénomènes humains apparemment régis par la liberté et l'indétermination - est la manifestation de l'ordre de Dieu. La mort est partie intégrante de cet ordre et ne se distribue absolument pas au hasard. Ainsi, la détermination de la valeur des rentes viagères, qui repose sur cet ordre de la mortalité, a partie liée avec la toute-puissance de Dieu et l'ordre divin. La question est donc une des clés de la manifestation de la Providence dans le monde. Illustration exemplaire de cette éthique protestante qui veut que les actions humaines a priori les plus engagées dans le monde ne soient en fait vouées qu'à la plus grande gloire de Dieu.

Dans le cadre restreint de ce travail - et la matière étant abondante nous nous sommes limité aux rentes viagères sur une seule tête et nous avons considéré les tontines comme des « rentes viagères en tontine », ainsi 
que l'on disait à l'époque, sans aborder, par exemple, les rentes sur plusieurs têtes ou les comparaisons auxquelles se livre Süßmilch pour déterminer ce qui serait, selon lui, le «meilleur système ».

Nous exposerons la «physico-théologie de la mortalité », pièce importante de l'ordre divin que prétend décrire Süßmilch; le problème général des rentes viagères; la notion d'espérance et de vie probable avec ce qu'elles impliquent en ce qui concerne les premières notions de l'assurance; enfin, le problème de la moralité des rentes et du type de contrat qu'elles instaurent entre l'individu et l'État, ce qui nous permettra de dégager les aspects philosophiques et sociopolitiques de la question.

\section{1. - LA PHYSICO-THÉOLOGIE DE LA MORTALITÉ}

\subsection{L'humanité : un régiment en marche}

«Pour expliquer cet ordre [celui qui règne dans les "changements de l'espèce humaine"] et les qualités qui lui sont jointes, on peut user de la marche d'un régiment. Si rien n'y est laissé indéterminé, si les soldats et les phalanges se suivent d'une même façon selon la taille, l'uniforme, l'arme et toutes autres choses, si les troupes sont également partagées, l'espace entre les phalanges et les troupes et la marche d'égale vitesse, et ainsi de suite, non seulement on y accordera de l'ordre, mais encore on y louera un grand ordre, et certes le plus grand, le plus accompli et le plus bel ordre ${ }^{1}$.»

L'ordre que Süßmilch croit et veut déceler dans les changements de l'espèce humaine est un ordre militaire, celui d'une armée en marche vers la victoire : celle de Frédéric II peut-être, et bien plus sûrement, celle des immortels qui partageront le royaume de Dieu.

Il convient d'abord de remarquer que l'image, outre la Vision de Mir$z a h^{2}$, provient en droite ligne de l'expérience de l'aumônier militaire, et

1. [Pour plus de précisions sur les références citées en notes, se reporter à la bibliographie finale, p. 415-417.] SüssmiLCH, 1761-1762, vol. I, chap. I, § 14, p. 52 (sauf exception dûment signalée, nous indiquons toujours la pagination des éditions en langue originale et nous sommes entièrement responsable de toutes les traductions qui figurent dans le présent article).

2. Le chapitre iv de l'ouvrage de James Bonar est consacré à Edmund Halley, non à son cuvre d'astronome, mais à sa contribution fondamentale à la démographie statistique, voir Halley, 1693. En voici un extrait, in Bonar, 1931, p. 123-125: "Nous avons tous une dette à l'égard du docteur William Farr pour avoir indiqué que les "Visions de Mirzah "d'Addison, dans le $\mathrm{n}^{\circ} 159$ du Spectator de 1711, sont une réminiscence de la table de survie de Halley. Addison, qui était né en 1672 (et qui mourut en 1719), pouvait fort bien, à l'époque de ses vingt ans, avoir eu vent de la parution de la Table en 1693. Beaucoup d'entre nous, avant ou après nos vingt ans, ont lu "les Visions": le pont de cette vie avec ses soixante-dix arches, les sorts variés de ceux qui le traversent ou échouent à le traverser, le nuage noir à chaque extrémité, les trappes cachées disposées de manière serrée à l'entrée du pont, de sorte que des cohues de gens ne sont pas plutôt sortis du nuage que beaucoup d'entre eux tombent dans les 
plus exactement, car les termes précédents sonnent trop catholiques, de celle du Feldprediger, du prédicateur au champ de bataille que fut Süßmilch pendant l'invasion de la Silésie en 1741.

Il faut ensuite rappeler que la littérature, de l'épopée antique au roman contemporain de Süßmilch, fait - et comment en serait-il autrement dans le cours agité des affaires humaines? - largement place à la guerre. Le champ de bataille est le lieu du hasard et du destin, croisée des chemins de l'arbitraire et de la Providence : les dieux homériques, le coup de canon qui tua Turenne, la balle qui a son billet de Jacques et de son maître. Aussi nous semble-t-il que deux sortes d'images convergent ici : le champ de bataille et l'ordre des troupes, le désordre et le fracas des armes d'une part, la stratégie et la prévision de l'autre; et Dieu passe la revue des troupes. Certains sortent du rang, d'autres y entrent, la marche humaine se poursuit, tout comme, image quasi contemporaine de Süßmilch, dans le champ clos kantien, s'affrontent les doctrines de la métaphysique.

L'Ordnung der Sterblichkeit, l'ordre de la mortalité - l'expression des arithméticiens politiques qui signifie que le phénomène quelque peu terrifiant et sacré qu'est la mort peut au moins perdre une partie de son mystère - est cet ordonnancement par lequel Dieu, non seulement maintient tout l'édifice naturel, mais « veille encore particulièrement sur les habitants du globe terrestre et sur les continuels changements auxquels nous sommes assujettis ${ }^{3}$ ».

\subsection{Qu' est-ce que l'ordre?}

L'influence que Dieu exerce est "occulte mais indéniable 4 », et croire, plutôt qu'à « l'ordre une fois élu par la Providence ${ }^{5}$ », à « une contingence fortuite ${ }^{6}$ " serait un non-sens. "Tout est ici ordonnancé selon certains nombres et certaines proportions ${ }^{7}$. »

Qu'est-ce donc que cet ordonnancement?

L'ordre provient d'abord de la ressemblance et de l'uniformité de choses différentes qui se trouvent rassemblées ou qui se succèdent. Ensuite,

trappes, lesquelles se font de plus en plus clairsemées vers le milieu, mais se multiplient et se rapprochent vers la fin des arches que l'on voit encore entièrement. C'est un agréable commentaire de la Table de survie [...] Du pluriel du titre (Les Visions), on déduit qu'Addison semble avoir projeté une seconde vision. Il est clair pour tous ceux qui lisent la première d'un bout à l'autre qu'elle fut écrite pour l'édification morale et non dans un quelconque but scientifique. Chacun de nous, au cours des années, lutte pour passer telle ou telle de ces arches; souvenons-nous de notre fin ultime qui peut venir tôt ou tard. Les Visions sont pareilles à un mythe platonicien à la fin d'un dialogue, là où il est habituel qu'apparaisse la morale de l'histoire. "

3. Sússmilch, 1741, préf. de l'auteur, p. XX.

4. Ibid.

5. Ibid., p. XXI.

6. Ibid.

7. Ibid., p. XX-XXI. 
lorsqu'une grande variété de choses est déterminée de façon analogue et que rien n'est laissé au hasard ${ }^{8}$, on se trouve devant le meilleur ordre alors possible. De plus, l'ordre qui concourt à la réalisation d'un but, s'il l'atteint constamment, est dit beau et parfait. On le constate avec plaisir et admiration. Enfin, cet ordre est constant et général. Il y a des exceptions, mais tout revient vite au train accoutumé.

Le recours aux grands nombres nous montre à la fois la régularité et la variabilité : les changements de l'espèce humaine s'opèrent dans l'ordre. Sans avoir rien à faire avec le hasard ou la nécessité naturelle, l'ordre est néanmoins contingent à nos yeux puisqu'il est l'œuvre du seul choix de Dieu.

De cet ordre optimal se déduit, non seulement l'existence de Dieu, mais encore sa providence comme loi constante.

Süßmilch, pour l'exposé de ces considérations, emploie volontiers le ton de la prédication:

«Et, non seulement, de cet ordre, se laisse déduire l'existence du Créateur le plus sage et le meilleur, mais il nous fournit encore nombre des plus hautes raisons pour en conclure la persévérante Providence du plus sage des Créateurs et sa prévoyance particulière pour l'espèce humaine ${ }^{9}$."

Et plus loin :

« Et qu'est-ce qui maintient toute chose dans ce bel ordre et dans cet équilibre? Ne nous faut-il pas nécessairement conclure, de cette conservation qui nous est inconcevable, à la main du gouverneur caché de toute chose qui se préoccupe de nous, non seulement lors de la Création, mais encore constamment par la suite ${ }^{10}$ ?"

C'est le Dieu tout-puissant et perpétuellement agissant. Il se montre un Arithmeticus infini et précis qui détermine tout ce qui, dans la nature et dans le temps, est en mesure, nombre et poids ${ }^{11}$. Mais, dans les petites

8. Unbestimmt gelassen, littéralement « laissé indéterminé ».

9. SUUsSMILCH, 1761-1762, vol. I, chap. I, § 17, p. 61.

10. Ibid., p. 63.

11. Cette sentence figure dans la préface d'une des « bibles » de l'arithmétique politique, à savoir L'Arithmétique politique ou Discours sur l'étendue et la valeur des terres... de William Petty. Voir PeTtY, 1670-1676, in trad. 1905, vol. 1, p. 268 : « La méthode que j'emploie [...] n'est pas encore très commune, car au lieu de me servir seulement de termes au comparatif et au superlatif et d'arguments purement rationnels [intellectual Arguments], j'ai adopté la méthode (comme spécimen de l'arithmétique politique que j'ai longtemps eue en vue) qui consiste à m'exprimer en termes de nombres, poids et mesures; à me servir uniquement d'arguments donnés par les sens [Arguments of Sense], et à ne considérer exclusivement que les causes qui ont des bases visibles dans la nature; je laisse à la considération des autres, les arguments qui dépendent des idées, des opinions, des désirs, des passions variables des individus [...] » Ce qui n'en correspond pas moins à cet endroit de l'Ecriture (Livre de la sagesse, XI, 21): «Sans cela même [sans l'envoi d'épouvantables et cruelles bêtes] ils [les peuples iniques] pouvaient périr d'un seul souffle, persécutés par leurs propres crimes, et renversés par 
choses, tout semble aller en désordre. Il faut donc rassembler nombre de cas singuliers, et sur beaucoup d'années, pour porter à la lumière les admirables et secrètes règles de l'ordre :

« Mais ne pouvons-nous pas avoir l'espérance que nous serons à même de juger justement de tout et de pénétrer l'enchaînement de tout, lorsque nous serons à même de pénétrer tous les petits cas dans le monde suivant toutes leurs circonstances, et de tout porter à la lumière? Alors sera montré que tout dans le monde s'accorde entièrement à la sagesse, à la bonté et à la justice de Dieu, et que tout a été légiféré par Dieu en une liaison telle que, pour sa gloire, il soit sans fin manifeste que ce monde est le meilleur que l'entendement infini a choisi entre tous les possibles. Mais si nous ne le pouvons dans les présentes ténèbres, soyons du moins contents de pouvoir espérer atteindre la lumière de la divinité, là où notre œil raffermi embrassera peu à peu l'enchaînement de toutes choses ${ }^{12}$.»

\section{L'ordre divin exclut par conséquent tout hasard :}

"Aucun homme raisonnable ne se laisserait aller là-dessus au rêve d'une nécessité ou d'un accident fortuit, attendu que particulièrement un hasard ne peut du tout exister là où l'on a quelque 100 cas dans lesquels se trouve toujours cet ordre, et, en revanche, presque pas un seul dans lequel se montre le contraire ( $\$ 43)$. C'est pourquoi cet ordre démontré fournit un nouvel exemple à l'aide duquel, selon les principes inébranlables de la métaphysique, on peut connaître, non seulement l'existence de Dieu, mais encore le gouvernement divin du monde [...] dès lors que l'on peut incontestablement faire voir à partir de principes métaphysiques qu'aussi peu cet ordre-ci que d'autres ordres contingents dans le monde ne peuvent provenir d'un pur hasard, et qu'en revanche ils nous persuadent de l'existence de Dieu et de ses sages desseins ${ }^{13}$.»

Le père de l'arithmétique politique, John Graunt, écrit que « les Hommes ne meurent pas selon des proportions exactes ni en fractions ${ }^{14} »$. La question est de savoir si, afin d'étudier la mortalité, on « feint » des hypothèses a priori ou si l'on étudie des données pour en tirer a posteriori des rapports. C'est la seconde voie que préconise Süßmilch. Mais, pour le pasteur, la méthode expérimentale s'inscrit toujours dans un cadre théologique et philosophique préalable, celui de l'ordre décrété par Dieu : aussi l'expérience n'a-t-elle pour rôle que de certifier l'existence de cet ordre aux yeux des hommes de peu de foi. Pour le théologien, cette entreprise n'est que de

le souffle de votre puissance; mais vous réglez toutes choses avec mesure, avec nombre et avec poids. "

12. Süssmilch, 1761-1762, vol. I, chap. I, $\$ 17$, p. 64.

13. Süssmilch, 1741, chap. v, $\$ 63$, p. 177-178.

14. GraunT, 1676, in trad. 1977, chap. XI, § 9, p. 105. 
vérification : l'ordre est présupposé. Cet ordre général — et l'ordre de la mortalité, un de ses avatars - conditionne la pratique des rentes viagères.

\section{2. — QU'EST-CE QU'UNE RENTE VIAGÈRE? LA « VIE PRÉSOMPTIVE 》}

\subsection{Des choses aussi curieuses qu'utiles}

«La précédente investigation et l'examen des morts suivant les âges ne sont pas moins curieux qu'utiles. C'est pourquoi Graunt a déjà fait l'essai de déterminer les morts et les vivants à chaque âge. Mais, après lui, le célèbre Edmund Halley a examiné beaucoup plus précisément la chose, attendu qu'elle en vaut la peine, parce que la détermination des rentes viagères en dépend ${ }^{15}$. 》

Aussi curieuses qu'utiles: c'est l'expression même que Süßmilch employait dès la dédicace ${ }^{16}$ de son ouvrage.

Il faut entendre qu'il s'agit de points qui sont dignes de la curiosité des savants mais aussi que la chose en elle-même est curieuse, pleine de surprises. L'utilité, toujours soulignée, indique que l'on entre dans le domaine des applications de l'ordre de la mortalité. Et cette utilité est immédiatement désignée comme la détermination des rentes viagères, sujet que Süßmilch avait évoqué en passant à propos de la mortalité des enfants ${ }^{17}$ et qu'il reprend plus loin ainsi :

«Le principal dessein dans lequel Monsieur Halley et Monsieur Struyck se sont beaucoup appliqués à cet examen de l'âge de l'homme est la détermination correcte des rentes viagères. Comme, en Hollande et en Angleterre, on fait grand négoce à ce sujet, il vaut bien la peine d'avoir quelque chose de précis en la matière [...] Mon but et l'espace dont je dispose ne souffrent pas que je me laisse aller à une dissertation de quelque étendue sur tout ce qu'il est nécessaire de savoir pour le calcul des rentes viagères [...] toutefois, je ne puis m'empêcher de faire ici état de ce que Monsieur Struyck a mis au jour, parce qu'il se trouve souvent des Allemands qui, en Hollande, ont à mettre de l'argent dans des rentes viagères ou qui ont envie de le faire ${ }^{18}$. "

Si Edmund Halley et Nicolas Struyck se sont appliqués à composer une table de survie, c'est principalement pour calculer les rentes viagères.

15. SÜssmilch, 1741 , chap. VI, $\$ 79$, p. 226.

16. Sússmilch, 1741 , dédicace à Frédéric II, p. IV : «En beaucoup d'autres choses, ces considérations ont pareillement une influence aussi curieuse qu'utile. » Halley parle, dans le titre de son Mémoire, des curious Tables de Neumann.

17. SüssmiLCH, 1741 , chap. vı, $\$ 70$, p. 206 : « On peut également juger par là à quel âge les parents peuvent prendre des rentes viagères sur leurs enfants, avec une certaine espérance en leur vie. "

18. Süssmilch, 1741, chap. vi, § 89, p. 245. 
Quoique Süßmilch ne maîtrise pas ce calcul en 1741 et qu'il ne puisse se «laisse[r] aller à une dissertation de quelque étendue», il ne peut « s'empêcher » de procéder à un développement sur ce sujet. La raison de cette apparence de paradoxe, Süßmilch nous l'indique clairement: en 1741 , le pasteur conçoit son ouvrage à la fois comme un manuel utile pour ses concitoyens et comme une illustration, par la démographie, du gouvernement de la Providence. La question des rentes viagères en est l'occa$\operatorname{sion}^{19}$.

\subsection{Le revenu d'une tête mortelle}

Une rente est un revenu ${ }^{20}$.

Le mot apparaîtrait au XII ${ }^{\mathrm{e}}$ siècle et serait issu d'un latin populaire ${ }^{\circ}$ rendita « ce que rend l'argent placé ». Rente prend très tôt la valeur spéciale de "revenu donné périodiquement en retour d'un capital aliéné ».

Émile Littré parle de revenu annuel :

«Dans le sens primitif et propre, rente est absolument synonyme de revenu annuel. L'usage y a introduit cette différence que rente se dit surtout des revenus que procure un bien-fonds ou une somme d'argent placée, et uniquement du revenu annuel que l'État paye à ses créanciers. De plus, revenu aujourd'hui comprend tout ce qu'on reçoit, tandis que rente ne comprend pas ce que l'on gagne actuellement par son travail ${ }^{21}$."

Donc la rente est un revenu particulier puisque provenant du produit de l'argent ou, comme diront les économistes, de la valeur d'un bien possédé et qui rapporte. La rente s'achète et se vend à un certain prix. C'est la détermination de ce prix qui constitue la question.

Le mot "viager " est dérivé au XIv siècle d'une forme féminine qui serait attestée au xiI dans le nord de la France, laquelle dérive elle-même de l'ancien français «viage», du mot «vie» suffixé en -age : le mot «viage » signifiait «cours, durée de la vie» et était également employé

19. Ce pourquoi le traitement de la question des rentes viagères est annoncé dès Sússmilch, 1741 , préf., p. XXXIII-XXXIV : «Que ce traité soit indispensable dans un pays où l'on négocie beaucoup de rentes viagères, c'est ce qu'ont assez montré ces deux grands hommes, Halley et Struyck, par leur difficile labeur dont j'ai rapporté ici le principal. »

20. Ce n'est qu'en 1761-1762, et non en 1741 où il ne le fait jamais, que Süßmilch donnera une définition exacte des rentes amortissables et des rentes viagères, voir SüssMILCH, 17611762 , vol. II, chap. xxIII, $\$ 493$, p. 367-368 : «Les rentes, que les Anglais nomment Annuities, sont principalement de deux sortes. Il y a 1) certaines rentes annuelles fixes qui consistent dans le revenu annuel du capital avec les intérêts à une époque déterminée [...] 2) L'autre sorte s'appelle "Rentes viageres", Leibrenten, Vitalitium, Annuities upon lives. On y comprend celles qui sont versées tout le temps de la vie d'une personne, que la personne meure bientôt ou qu'elle devienne très vieille. La première sorte de rente a son fondement dans une convention de libre volonté. Mais la raison déterminante des rentes viagères réside dans la durée de la vie d'une personne, et dépend donc de l'ordre de la mortalité et non de la libre volonté des contractants. »

21. LITTRÉ, 1877 , vol. 5 , p. 5440. 
dans le domaine juridique pour signifier « usufruit à vie », puis au début du $\mathrm{XVII}^{\mathrm{e}}$ siècle pour « personne qui jouit d'une rente pendant sa vie ». L'adjectif s'applique à une personne qui bénéficie, sa vie durant, de ressources. Le sens est sorti d'usage. Le mot s'emploie spécifiquement pour qualifier une rente, une pension qui doit durer pendant la vie d'une personne et non audelà. « Viager » désignait encore en 1771 un rentier à vie. L'acception a disparu. Un viager correspond à un revenu viager.

Ainsi, dans le cas de la rente viagère, le bien que l'on possède est, tout bonnement, la vie, le fait de vivre. On constitue une rente « sur la tête » de quelqu'un. Dans l'allemand d'aujourd'hui, "rentes viagères » se dit lebenslängliche Renten et, chez Süßmilch, nous trouvons Leib-Renten. La formation de ce dernier mot est intéressante. Leib veut dire corps ou tronc sans membres, ou encore panse, bas-ventre, carrure d'habit, et l'on dit Leib und Leben wagen pour " hasarder sa vie ». Au xIII siècle, en Italie, le capital est le corpo, le corps. Intérêts sur le corps ou le capital, c'est la vie même qui est en jeu, la vie terrestre, celle du corps mortel engagé dans le risque de l'échange économique : il s'agit bien d'une rente sur le corps tant que ce dernier est encore en état de marche.

Quel en est le principe? Une rente viagère est un revenu versé, sa vie durant, à une personne - le " crédirentier » - par une autre - le « débirentier »- à laquelle la première a cédé un capital. Prenons ce que l'on appelle la rente amortissable ou rente à terme fixe. Supposons que je possède 1000 francs : avec cette somme, je me propose d'acheter une rente pour vingt ans. Je cède ces 1000 francs à une autre personne - qui peut être une caisse, une banque, l'État, etc. - qui me versera la rente périodiquement pendant vingt ans. Cette période est préalablement et contractuellement définie. Par conséquent, je vends mes 1000 francs, mon capital, à un acheteur qui me le reverse avec intérêt. L'acheteur peut utiliser ou faire fructifier immédiatement le capital ainsi constitué. En ce qui me concerne, je suis assuré d'un revenu fixe pendant vingt ans. Le calcul se fait à partir du capital que j'ai versé, du taux d'intérêt et de la durée des versements périodiques de la rente. On parle de terme de la rente pour chaque versement de celle-ci. La rente peut être versée dès la signature du contrat ou à la fin de la première année, ou au début de la deuxième, c'està-dire de la première année pendant laquelle court le contrat, ou encore après un délai convenu d'un commun accord entre les parties.

Mais le problème est autre lorsqu'il se pose ainsi : je désire bénéficier d'une rente viagère de 100 francs par an : quel capital dois-je engager pour l'acheter? Combien moi, crédirentier, dois-je verser au débirentier pour que ce dernier me constitue en contrepartie une rente annuelle de 100 francs et ce jusqu'à ma mort?

Or, que sait-on du terme de sa vie? Comment fixer le prix d'achat? Il n'y a qu'un moyen de conclure un contrat qui satisfasse les deux parties : 
estimer ce qui me reste de vie, c'est-à-dire faire entrer en compte la durée probable de vie qui me reste. Nous ne sommes plus dans le seul calcul financier du jeu des intérêts composés — " l'intérêt et l'intérêt de l'intérêt » comme on dit à cette époque - mais dans ce que l'on appelle de nos jours le calcul actuariel, c'est-à-dire l'estimation d'une rente aléatoire.

Au moment où le contrat est signé, ni moi crédirentier ni mon débirentier ne savons combien de temps il me reste à vivre : ce peut être dix minutes, un an, cinq ans ou cinquante. Il s'agit d'un jeu, d'un pari sur la vie qu'il me reste à vivre, ce que Leibniz appelle la vie présomptive 22 .

Mais chaque durée qui peut me rester à vivre, et ce, en fonction de mon âge présent, est plus ou moins probable. D'où la question : n'est-il pas possible d'estimer raisonnablement ma vie présomptive, de sorte que le débirentier ne fasse pas faillite et que je ne sois pas condamné à perdre à tous coups? Voici la solution proposée par Leibniz :

«Demonstration exacte, que la moyenne longueur de la vie humaine est de 40 ans, et qu'une pension viagere achetée pour un enfant né depuis peu doit estre jugée equivalente à une pension de 40 ans. Par là nous trouverons aisément la moyenne longueur de la vie humaine. Car un de ces enfans susdits estant consideré en son particulier, il y a autant d'apparence à dire qu'il mourra dans la premiere, ou dans la seconde, ou dans la troisième année, que dans quelque autre jusqu'à la quatrevingtunieme [...] Donc nous pouvons dire que 40 ans sont la longueur moyenne de la vie humaine. Et par consequent s'il y avoit une rente à vie ou pension viagere affectée à un enfant né depuis peu, il la faudroit considerer comme si c'estoit une pension temporelle fixée au nombre de 40 ans, les quels écoulés elle devroit expirer, et par là on peut estimer sa valeur presente, c'est à dire pour combien une telle pension devroit estre achetée presentement, ayant égard au rabat, comme j'ay fait voir ailleurs $[\ldots]^{23}$ »

Mais c'est une solution théorique qui ne tient évidemment pas compte de l'ordre réel, « conforme à l'expérience », de la mortalité. Il n'en est pas moins vrai que Leibniz donne ici les principes du calcul de la vie moyenne.

22. LeIBNIZ, 1680a, in trad. 1995. L'expression « vita praesumtiva » se trouve p. 310 . Voir, également, LeIBNIZ, 1680b et 1680c, in Berlin, 1986, " anni praesumtivi ", p. 449, " vita hominis residua praesumtiva ", p. 452. Rappelons d'abord que ces manuscrits ne furent connus qu'au XIX ${ }^{e}$ siècle et que, bien entendu, Süßmilch en ignorait jusqu'à l'existence. Marc Parmentier, dans son commentaire, insiste à juste titre sur la valeur juridique de l'expression. On peut également remarquer sa connotation probabiliste : la durée sur laquelle il serait raisonnablement (Süßmilch écrira «vernünftig ») possible de parier. Voir dans LeIBNIZ, 1680d, in Berlin, 1986, la « supposition fondamentale » au sujet des 81 enfants, p. 459 : "Enfin comme nous les [les 81 enfants nés depuis peu] avons supposés tous d'une egale vivacité, ce sera comme si on tiroit au sort, pour sçavoir le quel doit mourir le premier, le second, le troisieme, et ainsi de suite, puisqu'il n'y a pas plus d'apparence ou raison pour l'un que pour l'autre. " Voir ROHRBASSER, VÉRON, 1997.

23. LeIBNIZ, 1680d, in Berlin, 1986, p. 459-460. 
Posé par rapport à l'estimation de ma propre vie présomptive, le problème est insoluble. Mais si l'on possède des données sur la survenue du décès d'un nombre suffisamment grand de personnes, on peut estimer que je suis un cas « statistique " parmi ce grand nombre et que ma vie présomptive peut être affectée d'une probabilité. C'est ce qui rend possible la pratique des assurances sur la vie, pratique qui se détachera, mais seulement peu à peu, du jeu ou du pari. Lorsqu'on dispose de données nombreuses, le risque devient, en moyenne, calculable. L'État, principal débirentier, y trouve son compte par l'application de la loi des grands nombres.

Nous voyons comment cette question est liée à la démographie et au calcul des probabilités : seule une table de mortalité correctement établie permet le calcul correct des rentes viagères. Et c'est précisément à l'époque de Süßmilch que s'élaborent les notions de durée probable et durée moyenne de la vie, de probabilité de survie et de ce qui deviendra l'espérance de vie. Si nous ne pouvons ici retracer, fût-ce à grands traits, l'histoire de ces notions ${ }^{24}$, nous pouvons du moins tenter de préciser le sens et la manière dont Süßmilch les comprend et les expose.

\section{3. - espérANCE, VIE PROBABLE, ASSURANCES ET PROVIDENCE}

\subsection{Vie probable}

C'est dans le cadre de la probabilité que vont être menées ces recherches sur l'ordre de la mortalité.

«[...] je ferai part au bienveillant lecteur de quelques observations sur les années et les âges différents auxquels les hommes meurent. Or plusieurs choses se trouvent derechef ici qui ne sont point encore prouvées à l'aide d'exemples suffisants, mais qui, ce nonobstant, sont au moins probables [wenigstens wahrscheinlich ${ }^{25}$.»

24. Une telle histoire, dans toute sa complexité et suivant des intrications telles qu'elle ferait se croiser l'histoire du calcul financier, du calcul actuariel, des assurances, de l'épargne, des problèmes démographiques et politiques afférents, sans oublier la naissance des idées de prévoyance à partir de celle de providence, une telle histoire n'existe pas, à notre connaissance. Parmi les ouvrages de référence, nous pouvons indiquer Pearson, 1977, une mine inépuisable dans laquelle nous avons largement puisé. On trouve une belle analyse du problème technique en ses origines mêmes dans HALD, 1990. La question des tables de mortalité est clairement exposée in BéHAR, 1976. Il faut également signaler le recueil de THIVEAUD et al., 1989-1990, utile mise au point sur le domaine français. Enfin, DuPÂQUIER, 1985a, et DuPÂQUIER, 1996, sont indispensables pour saisir l'enjeu de la question.

25. Sússmilch, 1741, chap. vi, $\$ 66$, p. 191. On notera l'analogie de ces propos avec la préf. de Christian Wolff à cette première édition, in ibid., p. 10-11: «En effet, hors la connais- 
Le terme allemand wahrscheinlich peut se traduire de deux façons : « probable » mais aussi « vraisemblable » comme le dit si bien la formation du mot : es scheint daß es wahr sei, c'est-à-dire «il semble que ce soit vrai », «c'est semblable à la vérité ». Ce n'est peut-être pas l'entière vérité, mais cela lui ressemble. Aussi, le probable est-il ici une manière d'approcher la vérité. Et l'on voit bien que c'est le manque d'exemples en nombre suffisant qui fait le «seulement probable ». Pour Süßmilch, la vérité n'est pas inaccessible, mais réside dans l'ordre divin. L'homme de foi en perçoit les traces. Elle existe, nous la connaissons, seuls manquent les exemples pour en persuader les incroyants. Les exemples ne sont là que pour mesurer la provisoire méconnaissance - et non ignorance - humaine. Le débat sur la mortalité et sur les rentes viagères se place tout entier dans cette confiance accordée au suprême géomètre ${ }^{26}$.

Puis, Süßmilch aborde la question de la durée probable de la vie :

« Par les précédentes tables, on peut ensuite également connaître les différents degrés de la mortalité ou, bien plus, de la vitalité humaine. Si, dans une loterie, il n'y a que 2 billets perdants contre une bonne chance, mon gain est plus probable que s'il y a 4 billets perdants, mais plus probable encore que s'il y a 20 cas pour échouer. Et s'il y a autant de billets gagnants que de perdants, la perte et le gain sont également probables, la crainte est aussi grande que l'espérance ${ }^{27}$."

Contrairement à beaucoup d'autres, ce passage n'est pas traduit de Halley. De manière caractéristique, il y est fait appel à la loterie, ce que fera Antoine Deparcieux ${ }^{28}$, et ce qu'avaient fait Jan

sance certaine, laquelle est certes à préférer partout où l'on peut l'obtenir, il en est aussi une probable qui, dans les affaires humaines, tient avec avantage lieu de certitude. Et la sagesse, qu'il nous faut montrer en tous les cas, réclame que nous nous y efforcions. Mais les théories vraisemblables ne peuvent pas toutes être obtenues par cette même voie par laquelle sont formées les théories certaines, savoir à l'aide de la démonstration, et il se trouve, dans la probabilité, bien des cas à méditer dont on peut prétendre juger ou par des raisons internes ou par des externes. "

26. Laquelle n'est pas sans rappeler le fameux «Dieu ne joue pas aux dés », symbole de cette confiance ainsi que de ce fait que les causes inconnues ou les « variables cachées » sont l'autre nom du hasard, lequel est impuissant à détourner le croyant de sa foi en la Providence. D'ailleurs, dans l'optique du pasteur (qui est également celle de Leibniz), même si Dieu jouait aux dés, il ne jouerait pas contre l'espèce humaine.

27. Süssmilch, 1741 , chap. vi, $\S 85$, p. 238.

28. Deparcieux, 1746. Le mathématicien français présente la probabilité de survie sous la forme généralisée du pari, p. 52-53 : «On peut, par exemple, parier 726 contre 8, ou $901 / 4$ contre 1, qu'un Rentier de l'âge de 30 ans, ne mourra pas dans l'espace d'un an. Car on peut supposer qu'il est un des 734 Rentiers vivans à l'âge de 30 ans : or sur ces 734 Rentiers vivans à l'âge de 30 ans, il y en aura 726 qui feront gagner, \& 8 qui feront perdre [...] On peut par la même raison parier 622 contre 112 , qu'un Rentier de l'âge de 30 ans vivra encore à l'âge de 45 ans; $\&$ il y a un contre un à parier, ou environ, qu'il vivra jusqu'à l'âge de 67 ans, parce qu'à cet âge il ne reste qu'environ la moitié du nombre des Rentiers vivans à l'âge de 30 ans. 
De Witt ${ }^{29}$, Christiaan et Ludwik Huygens ${ }^{30}$ et Nicolas Struyck ${ }^{31}$. La probabilité est envisagée sous l'angle du pari, et la question est bien de trouver combien de temps on peut parier que tel individu vivra encore ${ }^{32}$.

La mort est assimilée à un Niete, soit à un «billet blanc », c'est-à-dire un billet qui n'attribue aucun gain. La continuation de la vie est un gutes Looß, soit un bon lot, un bon sort. Süßmilch explique dans quelles conditions la chance de gagner peut être plus grande, ou encore les conditions dans lesquelles il est plus ou moins avantageux de jouer. Vie et mort assimilées à une grande loterie : voilà l'héritage d'une des origines, la plus fameuse, du calcul des probabilités à savoir la réflexion sur les jeux. Dieu en personne est l'organisateur de cette loterie puisqu'il s'agit d'un équilibre, d'une balance entre la crainte et l'espérance ${ }^{33}$.

De cette espérance naît la notion de vie probable. Süßmilch suit de près le texte de Halley ${ }^{34}$ :

«Il est également facile de trouver le moment où le pari devient égal, ou celui où les raisons qui font qu'une personne vivra ou mourra sont égales. Ou encore si l'on compte cent personnes qui auraient toutes le même âge et que l'on demande après combien d'années la moitié d'entre elles seraient mortes, on peut trouver la réponse de cette manière : on divise par deux le nombre de ceux

Celui qui parieroit sur tous séparément, gagneroit encore autant d'un côté, qu'il perdroit de l'autre. 》

29. De WrTT, 1671, in DupÂQuIER, 1985b. La probabilité de survie est exposée dans les trois présuppositions de De Witt, p. 361-363. Voir, également, DuPÂQUIER, 1996, chap. II, p. 21-32, et HALD, 1990, p. 122-131.

30. Huygens, 1669, in La Haye, 1895. Voir Dupâquier, 1996, chap. III, p. 33-46 et Hald, 1990, p. 106-110.

31. StruYCK, 1740, in StruYck, 1912, p. 232-233 : « Supposons par exemple qu'on considère 100 personnes du même âge et qu'on demande à quelle époque la moitié seront mortes; car alors, s'il s'agit de personnes en bonne santé ou telles au moins qu'on pourrait les assurer, il y a une chance contre une qu'une personne déterminée est encore en vie [Suit un tableau des vies probables] On voit par ce qui précède qu'on peut parier 1 contre 1 qu'un homme de 50 ans vivra encore $131 / 2$ ans [...]”

32. Nous verrons toute l'importance de cette question, infra p. 405-413, lorsque nous étudierons la moralité des rentes.

33. Süssmilch, 1741, chap. vi, $\S 67$, p. 194-199, présente une table de survie, à partir de laquelle il entreprend son étude de la mortalité et tire les premières conclusions suivantes, in ibid., § 69, p. 204-205 : « Si l'on avait davantage de telles listes spéciales pour davantage de villes et aussi pour la campagne, on pourrait en déterminer beaucoup de belles choses [...] On pourrait déterminer combien d'enfants restaient et vivaient à chaque âge, ainsi que le degré de la mortalité ou de la force vitale à chaque âge, tout comme l'espérance que l'on pourrait avoir qu'ils vivraient encore, et ainsi de suite. » Outre la demande de données toujours en plus grand nombre - qui, seules, peuvent permettre au principe des grands nombres d'être opératoire - SüBmilch introduit ici les deux concepts fondateurs de l'ordre : le degré de la mortalité (der Grad der Sterblichkeit) ou de la force vitale (Lebhaftigkeit) à chaque âge, et l'espérance de vivre (die Hofnung, dans l'orthographe du pasteur). La notion de degré servira à l'étude des années climatériques. Celle de l'espérance conditionne le traitement du problème des rentes viagères.

34. SÜsSMILCH, 1741, chap. vI, § 85, p. 239-240. 
qui vivent à l'âge donné et l'on cherche dans la table où se trouve cette moitié, cet endroit est alors l'âge qu'une personne peut encore raisonnablement se promettre d'atteindre. Une personne qui a 30 ans peut espérer ainsi qu'elle vivra encore 27 à 28 ans. Jusque-là, la crainte de la mort est égale à l'espérance de la vie. Mais ensuite, la crainte l'emporte sur l'espérance. Cependant, un homme raisonnable usera-t-il, ou peut-il user de cela comme d'une certitude, là où il peut se trouver aussi facilement sous terre que dessus ${ }^{35}$ ? »

L'hypothèse est bien qu'il y a un moment dans la vie où l'on a autant de chances d'être mort que d'être encore vivant : la vie probable est donc à proprement parler le seul moment où les conditions d'équiprobabilité sont satisfaites et où le pari est équitable.

La vie probable est ainsi présentée comme une espérance raisonnable ou rationnelle [reasonably, vernünftig], c'est-à-dire de bon sens. Le bon sens et la raison résident dans le pari égal entre la crainte et l'espérance, les deux grandes catégories de la morale stoïcienne. Il s'agit d'une balance entre deux sentiments. Jusqu'au moment où intervient l'espérance de vie, l'espérance l'emporte sur la crainte. Après, la crainte à son tour l'emporte. Ce moment de la parfaite indifférence est donc bref, et la vie est bel et bien assimilée à une balance dont la position d'équilibre est précaire. Mais la morale luthérienne encourage cette absence d'indifférence. Lorsque l'espérance l'emporte sur la crainte, il faut se réjouir. Et lorsque la crainte l'emporte sur l'espérance, il faut se préparer.

Il est intéressant de voir que la réflexion qui termine cet alinéa, et qui ne doit rien à Halley, est encore toute pénétrée de la subjectivité que n'abandonne guère le pasteur : ce moment de l'équilibre ne représente jamais une Sicherheit, une sûreté ou une sécurité. L'individu est sujet de son seul Dieu et non des règles de la probabilité qui ne valent que pour l'ensemble.

C'est le ton de la prédication que nous allons rencontrer en conclusion des réflexions de Süßmilch sur la vie probable. Le prétexte du sermon se trouve encore une fois chez Halley :

« Outre les usages mentionnés dans mon précédent [article], il ne sera peut-être pas inacceptable d'inférer des mêmes Tables combien est injustifiée notre manière de nous plaindre de la brièveté de nos Vies et de nous estimer dupés lorsque nous n'atteignons pas un Âge Avancé; il appert [des tables] que la

35. Halley, 1693a, p. 602 : «Use III. But if it be enquired at what number of Years, it is an even Lay that a Person of any Age shall die, this Table readily performs it: For if the number of Persons living of the Age proposed be halfed, it will be found by the Table at what Year the said number is reduced to half by Mortality; and that is the Age, to which it is an even Wager, that a Person of the Age proposed shall arrive before he die. As for Instance : A Person of 30 Years of Age is proposed, the number of that Age is 531, the half thereof is 265, which number I find to be between 57 and 58 Years; so that a Man of 30 may reasonably expect to live between 27 and 28 Years. " 
moitié des nés sont morts au moment de la Dix-septième année, puisque, à ce moment, 1238 sont réduits à 616 . Dès lors, au lieu de murmurer contre ce que nous appelons une Mort intempestive, nous devons, avec Patience et sangfroid, nous soumettre à cette Dissolution qui est la nécessaire Condition de notre Matière périssable, et de notre Organisation et Composition belles et fragiles : [et nous devons] compter pour une Bénédiction d'avoir survécu, peutêtre de plusieurs Années, à cette Période de la Vie à laquelle la moitié de toute l'Espèce Humaine ne parvient pas ${ }^{36}$. »

Il n'est ici fait aucune allusion à Dieu ni à sa providence, mais à la dissolution de notre perishable materials et de notre frail structure. Il s'agit de la mort du corps, de la nécessaire et naturelle soumission de la matière à l'ordre des choses sublunaires, celles qui connaissent la génération et la corruption propres à leur nature.

De ce matériau, «brut » en son genre, Süßmilch tire une envolée de sermon :

« Enfin, Monsieur Halley conclut encore de sa table qu'il serait injuste de se plaindre de la brièveté de notre vie, et de croire que ce soit une iniquité de ne point atteindre un âge avancé. Oui, il appert de ce qui précède qu'une moitié des nés meurt dès avant la septième année et certes encore auparavant $(\$ 71)$. $\mathrm{Au}$ lieu de murmurer contre la mort précoce, on doit bien plutôt remercier Dieu de sa miséricorde, lequel nous permet de survivre à telle période de la vie à laquelle une moitié de l'ensemble de la souche humaine ne parvient pas. À cette réflexion j'ajoute encore celle-ci, qu'un chacun se doit équitablement faire en observant la quantité de ceux qui meurent avant d'avoir atteint un âge mûr et viril : puisqu'il plaît à la divine providence de parsemer le chemin de la vie de tant d'obstacles et qu'il faut que tant d'hommes partent avant qu'ils n'aient atteint l'âge et la situation qui leur permettent de servir droitement Dieu et leur prochain, c'est que Dieu, dont la sagesse ne choisit rien sans raison suffisante, doit nécessairement avoir eu des desseins particuliers pour quoi il te destine, toi, à vivre. Il faut par là qu'un homme soit encore plus encouragé à remplir, autant qu'il lui est possible, les desseins généraux et particuliers de son Dieu. Et comme il doit se considérer, entre beaucoup, pour ainsi dire comme acheté par la mort, que cela lui soit bon pour rembourser en tout temps et occasion, afin de se conduire conformément aux sages et bons desseins de son Dieu ${ }^{37}$. »

Süßmilch ramène donc l'ordre de la survie aux raisons suffisantes qu'a Dieu de faire vivre ou non tel ou tel. Nous remarquons comment il s'approprie les paroles de Halley, puisque, au lieu de murmurer, il faut plutôt remercier Dieu de sa miséricorde : le premier membre est pris directement au texte de Halley, le second est le commentaire religieux de Süßmilch.

36. Halley, 1693b, in Westmead, 1973, p. 655.

37. Sùssmilch, 1741 , chap. vi, $\$ 87$, p. $243-244$. 
L'ordre général « descend » jusqu'à un ordre particulier dont dépend la vie des individus. Aussi chaque individu, du côté de la foi, est-il une pièce aussi importante qu'une autre dans le jeu général. D'où la possibilité de la statistique : chacun est égal aux yeux de Dieu quant à l'ordre ${ }^{38}$.

En outre - et c'est une indication qui nous semble intéressante dans un propos qui est consacré entre autres à des questions d'utilité pratique et financière - la durée de vie est présentée comme un contrat marchand où il s'agit d'achat et de remboursement. Dieu serait le grand emprunteur qui nous paierait en rentes d'années, capital que nous lui rembourserions - il nous l'a, pour ainsi dire, escompté - avec intérêt, par notre conduite conforme à ses desseins. C'est la clé de la réflexion du physico-théologien sur les rentes viagères. Süßmilch, jusqu'à sa mort, ne changera jamais de point de vue là-dessus.

\subsection{Rentes viagères et assurances sur la vie}

L'ordre divin consiste en ceci que Dieu, veillant sur chaque existence particulière, veille en même temps au bonheur de la collectivité et des institutions qui le régissent.

Le système des rentes touche aux finances d'un particulier mais également à celles de collectivités comme l'État ou certaines sociétés qui empruntent et reversent sous forme d'annuités ou de ce que l'on nomme des pensions. Au moment où écrit Süßmilch, nous sommes au commencement des systèmes d'assurances, comme en témoigne la quatrième utilité de la table de survie dans le Mémoire de Halley:

«Utilité IV. Par ce qui a été dit, le Prix d'une Assurance sur la Vie doit être réglementé, et la différence découverte entre le prix pour assurer la Vie d'un Homme de 20 ans et d'un de 50 ans, par Exemple; il y a 100 contre 1 [à parier] qu'un Homme de 20 [ans] ne mourra pas en l'espace d'une année, mais seulement 38 contre 1 pour un Homme de 50 Années d'Âge ${ }^{39}$. »

S'ensuit immédiatement la cinquième utilité :

« En dépend l'Évaluation des Rentes Viagères; car il est équitable que l'Acheteur n'ait à payer que la part de la valeur de l'Annuité [de la rente amortissable] proportionnelle aux Chances qu'il a de vivre $[\ldots]^{40}$.»

38. D'où l'emploi, très rare chez Süßmilch, du dich qui désigne bien tel individu particulier. Nous pensons naturellement à Matthieu, X, 29, 30 : « N'est-il pas vrai que deux passereaux ne se vendent qu'une obole? Et néanmoins il n'en tombe aucun sur la terre sans la volonté de votre Père. Et même les cheveux de votre tête sont tous comptés. »

39. Halley, 1693a, p. 602.

40. Ibid. 
Süßmilch, traduisant cette fois Struyck, consigne ce rapport entre « assurances sur la vie» et rentes viagères ${ }^{41}$. Au début du $\S 90$, Süßmilch emprunte ses exemples chiffrés à ce passage de Struyck :

"Ce qui précède [à savoir le calcul des rentes] permet aux directeurs des Maisons où l'on achète sa place pour toute la vie, de calculer aisément quelle somme doit être payée par une personne d'un âge déterminé. Supposons par exemple qu'une femme âgée de 48 ans veuille acheter une place dans une Maison où la pension complète durant un an est évaluée à fl. 300 . On demande la somme comptante qu'elle devra payer en entrant dans la Maison. La valeur de l'assurance pour une femme de 45 ans est de fl. 1140, et pour une femme de 50 ans de fl. 1020 [Struyck fait la règle de trois, puis] Pour obtenir annuellement fl. 80 à cet âge on doit payer fl. 1068 au comptant : il s'ensuit que pour dépenser fl. 300 par an, on doit payer fl. $4005^{42}$.»

\section{Struyck fait ensuite ce calcul :}

"Si l'on avait fait cette condition qu'au bout de 10 ans, si cette dame vivait encore et qu'elle ne désirait plus rester dans la Maison, elle pourrait en sortir et qu'on lui rendrait la somme à laquelle elle aurait droit, nous pouvons calculer cette somme $[\ldots]^{43}$.»

Le pasteur s'engage alors dans un plaidoyer pour l'implantation en Allemagne des établissements dont parle Struyck :

" Pourquoi de tels établissements ou de semblables ne sont-ils pas fondés chez nous? $\mathrm{Si}$, par exemple, de tels établissements étaient fondés dans une région de notre pays où les denrées coûtent peu, pour différentes sortes de gens, pour les deux sexes, aussi bien que pour les personnes faibles, ou les fous etc., ils viendraient beaucoup en aide à mainte famille. Un mari qui a été aux gages laisse souvent derrière lui une femme qui ne conserve de reste qu'à peine quelques centaines de thaler. Elle vit dans un endroit comme Berlin où les denrées coûtent cher, n'en est jamais sortie et ne sait donc où aller. Quand elle mettrait en sûreté son peu d'argent, elle ne pourrait nonobstant aller bien loin avec 30 ou 40 thaler par an; c'est pourquoi, surtout si elle est accoutumée à mieux, elle dépense tout et finit par tomber à la charge des autres. Mais s'il y avait de tels établissements dans des régions où les denrées coûtent peu, où la viande, le poisson, le pain, le bois et autres sont en telle abondance que l'on ne peut presque point faire argent d'eux, de telles personnes pourraient y vivre agréablement pour 30 thaler. Elles auraient de la compagnie et du plaisir à vivre là, et elles pourraient se l'acheter avec quelques centaines de thaler ${ }^{44}$.»

41. Süssmilch, 1741, chap. vI, § 90-91, p. 249-254.

42. StruYCK, 1740, in STRUYCK, 1912, p. 225.

43. Ibid.

44. Süssmilch, 1741, chap. vI, $\S 90$, p. 249-250. 
C'est le principe des caisses de veuves. Christian Jacob Baumann indique au début de son Traité ${ }^{45}$ que Süßmilch avait pensé à un mémoire sur cette question mais qu'il n'a pas eu le temps de l'écrire ${ }^{46}$.

Dans L'Ordre divin de 1761-1762, la création de ces établissements d'assurances est également liée à la nécessité d'encourager les mariages. C'est l'objectif principal de ce deuxième Ordre divin : tout subordonner au commandement mosaïque Croissez et multipliez et donc étudier, dans une perspective résolument populationniste, la croissance en nombre, commandée par Dieu selon un ordre, de l'espèce humaine :

«XII) À tout cela, j'ajoute encore les caisses de veuves comme moyen d'inciter au mariage. Elles sont particulièrement nécessaires aux personnes qui rendent des services à l'État dans des emplois tels qu'il leur est impossible de pouvoir mettre quelque chose de côté si elles ne veulent pas entamer leur loyauté. Et encore plus pour celles qui servent dans les grandes villes où tout est habituellement cher et où le luxe fait loi. Dans ces conditions, un grand nombre se détournent du mariage, et qui pourrait bien les en blâmer? En attendant, l'État y perd. Le préjudice est encore plus grand si ces personnes se laissent entrainer à la prostitution. Les mœurs en souffrent en général, et le service même de la patrie se trouve alors en danger. Mais si les employés voyaient venir ne serait-ce qu'un peu de subsistance et de secours pour eux et les leurs, ils se décideraient plus aisément à se $\operatorname{marie}^{47}$. "

Comment favoriser la fécondité générale et les mariages, telle est la préoccupation de ce onzième chapitre. Les mesures prises impliquent un rôle accru de l'État par rapport à 1741, un rôle d' 'État-providence ». L'œuvre de Dieu doit donc être secondée par celle des hommes. Süßmilch, fidèle à ses positions mercantilistes, indique que la multiplication de population, cette population qui constitue la rente, fait le revenu du souverain ${ }^{48}$. Süß-

45. BaumanN, in SÜssmilch, 1776, vol. III, p. 432-619.

46. BaUmanN, in Süssmilch, 1776, Abhandlung von Wittwenverpflegungsgesellschaften, $\S 1$, p. 432 : « Süßmilch avait promis $(\$ 250)$ de traiter également ce sujet dans ce chapitre, et d'insérer en particulier les avis et les calculs que Struyk a fournis là-dessus. » Le $\$ 250$ auquel Baumann fait allusion est, pour une grande part, une reprise de 1741. Voir SússmLCH, 17611762 , vol. 1, chap. XI, § 250, p. 486-487 : « Monsieur Struyck a donné, tout comme en général pour l'établissement d'autres caisses de veuves, de très bons renseignements là-dessus et il y a joint les calculs appropriés. Je ferai plus état de ces derniers ci-après, à l'occasion des rentes viagères. » La mention que Süßmilch fait de Struyck renvoie à STRUYCK, 1753, in STRUYCK, 1912, en part. au chap. v de la sixième partie "Remarques sur les pensions pour veuves ", p. 410-423. Ce chapitre contient surtout des calculs. Notre propos n'est pas ici de faire une comparaison avec le Traité (= Abhandlung, cit. supra) de Baumann : l'histoire de ces caisses, qui participe d'un projet beaucoup plus vaste, celui de l'histoire des rentes viagères, tontines et assurances sur la vie, n'a pas sa place dans le cadre nécessairement restreint de ce travail.

47. Süssmilch, 1761-1762, vol. 1, chap. XI, $\$ 250$, p. 484-485.

48. Par ex., Sússmilch, 1741, chap. vIII, $\S 115$, p. 345-347. 
milch fait également rapidement allusion à un taux croissant de la rente en fonction du revenu. Puis il ajoute cette mesure étonnante :

«3) la peine infligée aux vieux garçons, dont j’ai fait état ci-devant (\$233), si elle ne venait pas à la caisse de dotation des jeunes filles, pourrait être opportunément ajoutée, prise sur les employés qui auraient pu se marier de manière démontrée mais qui ne l'auraient pas fait par égoïsme ou pour d'autres raisons ${ }^{49}$."

Il est remarquable que Süßmilch imagine une contribution en argent de la part des vieux garçons, laquelle irait à une caisse de dotation des jeunes filles ou s'ajouterait à la rente de ceux qui ne se sont pas mariés par caprice ou pour d'autres raisons. Mais comment faire la preuve de ce «caprice» ou de ces autres raisons? L'État que propose Süßmilch est tout pareil à son Dieu : rien ne lui échappe et il sonde les reins et les cœurs. Il ne faut pas oublier que le pasteur a en tête la première République romaine avec ses Censores ou gouverneurs des mœurs : c'est l'ordre moral, voire théocratique, qui doit régner.

Enfin, et dès 1741 , Süßmilch préconise les monts de piété ${ }^{50}$ et se plaint de ce qu'il constate être un retard pour les États allemands. Il va ainsi dans le sens de ce qu'il sait sans doute être une des tendances de la politique future de Frédéric II : sortir la Prusse du féodalisme et lui donner des structures économiques précapitalistes. Sa conclusion est tout entière tournée vers ce projet :

«Le gain restant pourrait être transformé en un capital pour familles indigentes, ou bien pourrait être donné pour aider les établissements susmentionnés dans lesquels on achète sa subsistance, et ainsi de suite. Si tous les tontiniers étaient morts, les revenus pourraient être ajoutés aux bourses des étudiants et ainsi, les enfants de ceux qui ont eu part à la tontine devraient être avantagés par rapport à d'autres pauvres, et ainsi de suite. Si je me trompe en la matière et si je vais trop loin dans ces propositions, on me pardonnera aisément de tels défauts parce qu'ils proviennent d'une bonne intention, dès lors que j'aimerais bien aussi par là contribuer un peu au bien-être extérieur de mes concitoyens ${ }^{51}$."

Le lien est explicitement fait et l'« engagement » du pasteur expressément affirmé. Il s'agit de contribuer au bien-être matériel des concitoyens; et ceci, pour un pasteur luthérien, est également conforme à l'ordre divin.

49. SÚSSMLCH, 1761-1762, vol. I, chap. XI, $\S 250$, p. 485.

50. Qu'il appelle Lombards à cause des banquiers d'Italie du nord qui fondèrent ce genre d'établissements au XIII siècle.

51. Süssmilch, 1741, chap. vı, § 91, p. 254. 


\section{4. - LA MORALITÉ DES RENTES}

\subsection{Vices privés, vertus publiques}

Reposant sur l'ordre divin de la mortalité, il est impossible que les rentes viagères - si utiles au particulier et à l'État, mais simultanément pari sur l'espérance que l'on a de vivre - soient immorales. Il ne s'agit pas, en effet, de se substituer aux desseins de Dieu, mais bien, conformément au principe de la physico-théologie, de les mieux comprendre et d'y mieux obéir en en étudiant la nature, c'est-à-dire leur manifestation providentielle dans l'ordre du monde.

Il est clair que l'État lance des opérations viagères pour réaliser du profit $^{52}$. Quel est alors le type de contrat qui se noue entre l'État et le particulier? Et ce contrat est-il équitable, donc moral ? Süßmilch va amplement traiter le problème en 1761-1762 ${ }^{53}$.

En effet, au moment où écrit Süßmilch, l'usure demeure illicite et criminelle, tandis que la notion de contrat est solidement établie ${ }^{54}$. C'est sur ce pied que Deparcieux traite des rentes.

52. En ce qui concerne la tontine d'Amsterdam de 1671, par ex., l'emprunt fut lancé par la ville d'Amsterdam pour financer sa participation aux dépenses de guerre et, en l'occurrence, la guerre de Hollande, menée de 1672 à 1678 par Louis XIV afin notamment de ruiner le commerce hollandais. Une rente viagère fixe de 100 florins était proposée, à tous âges. C'était le prix de souscription qui variait avec l'âge du bénéficiaire. C'est ce prix de souscription que donne le tableau de Sússmilch, 1741, chap. v, $\S 65$, p. 189.

53. Süssmilch, 1761-1762, vol. II, chap. xxIII, § 514-516, p. 395-405.

54. L'aboutissement de cette sécularisation de l'intérêt peut, selon nous, être résumé par cette prise de position présente in [ANONYME], 1787, p. 159 (il s'agit d'une note ajoutée au chap. intitulé " De la Regle d'intérêt ») : «Il y a plusieurs occasions où il est licite de retirer les intérêts d'un argent prêté; c'est $1^{\circ}$. quand le prêt est cause qu'on souffre réellement un dommage, ou qu'on manque à faire un profit légitime; c'est ce qu'on appelle dommage naissant, \& lucre cessant. $2^{\circ}$. Quand l'argent que l'on prête est ordinairement employé dans le négoce, ou qu'il y est prochainement destiné. $3^{\circ}$. Lorsque les intérêts sont adjugés par Sentence du Juge, sur la demande que le créancier fait en justice de son principal \& de ses intérêts, pourvu que le créancier souffre du dommage par le retardement du paiement, ou qu'il y ait de la négligence de la part du débiteur. $4^{\circ}$. Dans les rentes constituées; parce que le sort principal est aliéné, \& qu'elles sont un véritable achat : ces intérêts se nomment arrérages. $5^{\circ}$. On peut, sans usure, entrer en société avec un Négociant, \& partager avec lui le profit qui doit résulter de son trafic, aux conditions requises dans les contrats de société, dont la principale est que chaque associé participe au gain ou à la perte. $6^{\circ}$. On peut demander caution pour assurance d'un argent que l'on prête; mais on ne peut prendre d'intérêts pour les risques que l'on court de n'être pas payé. L'usure est le profit que l'on exige en vertu du prêt sur ce qu'on a prêté à quelqu'un, en argent, en bled, en vin, ou en autre chose qui se consume par l'usage; elle est illicite \& criminelle. On peut quelquefois emprunter à usure ; mais sous cette condition : que celui qui emprunte le fasse pour sa propre nécessité, ou pour celle de son prochain, \& que cette nécessité soit très-grave. Louis XIV, par une Ordonnance de 1665, a fixé les intérêts au denier 20 , ou à 5 pour cent. » 
À vrai dire, la position de Süßmilch sur ce traitement apparaît dans une lumière quelque peu contrastée : nous assistons, en effet, à une diatribe morale dirigée contre les Hazardspiele, les jeux de hasard, diatribe uniquement destinée à défendre d'autant plus solidement la pratique des rentes viagères. Le jeu de hasard est, pour le pasteur, toujours sous le coup de la malédiction qui frappe le «sort diviseur » lorsque l'enjeu de ce que décide ce sort est vain. Même si c'est Dieu qui manipule les dés, la faiblesse humaine est trop grande pour ne voir là qu'une tentation : le jeu de hasard est une passion et, comme telle, condamnable. En revanche, la rente viagère - et son lien explicitement affirmé avec l'assurance - bâtie sur l'ordre de la mortalité tel qu'il est voulu par Dieu, facteur d'enrichissement pour l'État, puisque ce facteur favorise le développement de la population et que, par contrecoup, le développement de la population le rend encore plus rentable, n'est point pratique induite par le péché. Le jeu de hasard est un vice privé, la rente viagère une vertu publique. Le jeu de hasard est un instrument de chute individuelle, la rente un instrument du bien commun. Süßmilch est encore proche d'une théologie rigoureuse, celle du Dieu terrible de l'Ancien Testament qui ne distribue pas le sort n'importe comment, ni, surtout, en vain.

La question de la Moralität se développe encore, selon la technique de la prédication. En voici l'exorde :

« Pour conclure, je vais encore ajouter quelque chose sur la moralité de ces rentes. Je conclus de la justification de la chose que l'on trouve dans Monsieur Deparcieux qu'il faut qu'elle ait été attaquée et déclarée en France être quelque chose de coupable [sündlich]. Il semble que l'on doive nécessairement les avoir mises du côté des jeux de hasard [Hazardspielen]. Il accorde qu'on peut les nommer jeux ou loteries dans lesquelles on parie à qui vivra plus longtemps que l'autre et où le vendeur de la rente est le dépositaire de l'argent mis au jeu, tient les comptes pour les joueurs et paie les gains. Mais, dans ce jeu, ne gagne rien celui qui, pour ainsi dire, tient la banque; il ne vit également pas sur les dépenses des joueurs, au contraire, il va jusqu’à prendre sur lui la charge de payer les dépenses, et ainsi de suite ${ }^{55}$.»

Ainsi, le pasteur voit dans un passage de l'Essai de Deparcieux une justification [Rechtfertigung], une défense, une apologie des rentes viagères. Il ne cite pas la référence précise. Il s'agit de la « Remarque »:

«On doit conclure de tout ce qu'on a dit jusqu'ici, que les rentes viageres de quelque manière qu'elles soient faites, sont des Jeux ou Loteries, où l'on parie à qui vivra le plus ${ }^{56}$. "

55. SüssMilch, 1761-1762, vol. II, chap. xxIII, § 516, p. 395.

56. DeparcieuX, 1746, p. 122. 
La déclaration est nette; il s'agit bel et bien de jeux et de paris. Mais Deparcieux va aussitôt s'employer à distinguer ces jeux particuliers, à la fois selon leur enjeu et selon le type de contrat qu'ils impliquent. D'ailleurs, tout ce qui a été dit jusqu'ici est l'élaboration de l'ordre de la mortalité qui constitue, pour ainsi dire, l'assise scientifique de ce qui suit.

«Celui qui prend l'argent pour en payer la rente, doit être regardé comme le dépositaire de l'enjeu, qui se charge de faire valoir l'argent qu'on lui dépose, $\&$ de tenir compte aux Joueurs du bénéfice qu'il en tire, puisqu'il leur paye à la fin de chaque année une partie des capitaux, avec les intérêts des parties de capitaux dont il étoit resté débiteur au commencement de la même année ${ }^{57}$. "

Il s'agit donc d'un contrat équitable entre créditeur et débiteur dans lequel chaque partie épaule l'autre eu égard au profit : l'argent que l'un fait valoir profite à l'autre qui l'a prêté.

Deparcieux ajoute :

« À la plûpart des Jeux ou Loteries, l'avantage est pour celui qui tient le jeu, \& le desavantage pour les Joueurs. Ici c'est le contraire en tout. $1^{\circ}$. À tous les Jeux on s'expose à manquer souvent du nécessaire : ici on s'assure du bien pour le reste de sa vie. $2^{\circ}$. À tous les Jeux \& Loteries, l'argent est mort pour les Joueurs pendant tout le tems qu'il est hors de leurs mains : ici l'argent travaille ou rapporte au profit des Joueurs tant qu'il y a des fonds dans les coffres du Dépositaire, ou tant qu'il y a quelques Rentiers vivans. $3^{\circ}$. Enfin ceux qui ont permission de tenir ces tripots, de quelque espece qu'ils soient, vivent largement aux dépens des Joueurs, sur les fonds desquels ils prennent toujours une partie assez considérable, \& aussi hardiment que s'ils leur rendoient un service important : ici bien loin que le Dépositaire prenne aucun bénéfice sur le fonds des Joueurs, il se charge de payer tous les frais ${ }^{58}$. »

À la lecture de ce texte, il apparaît bien, selon nous, que ce que Süßmilch perçoit comme justification n'est que comparaison de type technique. Deparcieux ne défend pas les rentes viagères, il en résume le mécanisme et le compare à celui des jeux de hasard, afin de soigneusement distinguer celles-là de ceux-ci. Pour lui, il ne paraît faire aucun doute que les deux choses sont différentes et que les rentes représentent un avantage alors que les jeux sont plutôt la marque d'une sottise. Mais, à lire ce texte, il nous semble qu'aucun jugement de caractère moral n'y est porté. Deparcieux est un homme des Lumières, amplement cité dans l'Encyclopédie, pour qui il ne fait pas de doute que ces contrats sont licites et représentent des opéra-

57. Ibid.

58. Ibid., p. 122-123. 
tions de commerce qu'on a tout intérêt à pratiquer et à développer. Il ne lui vient pas à l'esprit de discuter du caractère éventuellement pécheur d'une pratique commerciale dont l'avantage financier ne fait aucun doute. Et sa conclusion nous semble le confirmer :

"Après cet examen il me semble qu'il faut vouloir être dupe, pour s'exposer aux Jeux de hazard, soit Dez, Roue de fortune, \&c. où il y a tant de désavantage, préférablement à un Jeu où il y a tout à gagner. C'est cependant ce qu'on voit tous les jours ${ }^{59}$.»

C'est le technicien des probabilités et du calcul financier qui conclut ici, un peu à la manière de ces vulgarisateurs d'aujourd'hui qui n'ont aucune peine à démontrer que la roulette ou le loto, en dépit des gains et des gros lots impressionnants qu'ils font miroiter, sont des jeux fort désavantageux au commun des mortels.

C'est exactement ce que dit Deparcieux : la raison conseille d'acheter des rentes, mais qui écoute la raison? On peut donc voir, en faisant parler cet implicite du texte, à l'extrême limite, une revendication de la faiblesse humaine face à l'infiniment plus forte attirance des passions, mais il nous semble que c'est déjà forcer ce qui est écrit.

C'est donc dans la vision des choses propre au pasteur qu'il faut chercher le prétexte des développements que nous allons lire. Il faut encore laver les calculs d'intérêt de la malédiction biblique que le pasteur voit dans le hasard et le prêt à intérêt : c'est bien Süßmilch — et non l'auteur de l'Essai - qui a à charge de montrer la toute-puissance de la Providence. Ses convictions lui montrent les rentes comme les voit Deparcieux. Mais quel homme serait assez insensé pour les voir ainsi sans voir en même temps que la mortalité est dans la main de Dieu?

"Es scheinet, daß man sie mit Hazardspielen müsse zusammen gesetzt haben » : pour le pasteur, les rentes viagères ne peuvent absolument pas être des jeux de hasard puisqu'elles reposent sur l'ordre de la mortalité qui, lui-même, repose sur l'ordre divin. Il s'ensuit que les rentes viagères participent de l'ordre divin, qu'elles sont une application de la manifestation de cet ordre et, pourquoi pas, une cause occasionnelle de l'intervention de la main de Dieu.

Deparcieux ne s'aventure jamais dans ces considérations. Or, la traduction que fait Süßmilch est très abrégée, résumée, au profit du commentaire qui est, lui, plus étendu.

La suite du § 514 affirme : « En fait, il n’y a pas même à examiner comment on pourrait vilipender la chose ou la déclarer coupable ${ }^{60}$.»

59. Ibid., p. 123.

60. SüsSMILCH, 1761-1762, vol. II, chap. xXIII, § 514, p. 395. 
Pour Süßmilch, la cause est entendue. Et l'on s'attend à ce qu'il traduise ou résume les arguments économiques employés par Deparcieux. Or, le pasteur fait un sermon sur la passion du jeu. C'est en accablant l'adversaire, le jeu de hasard taxé de tous les vices, qu'il procède à une apologie des rentes.

Dans la première partie du sermon, il justifie ainsi les rentes :

«Un homme à son aise place une partie de sa fortune dans les rentes afin d'obtenir, pour lui ou les siens, un profit autorisé. Celui qui met la $100^{\mathrm{C}}$ ou la $50^{e}$ ou la $30^{e}$ partie de son bien, ou encore si celui qui possède 10000 thaler met 100 ou 200 thaler dans une tontine, et en vérité pour gagner ou pour perdre, ou en répartit la $10^{e}$ voire la $5^{e}$ partie en rentes sur la tête de ses enfants pour le temps de leur vie et place ses fonds de manière à ne pas lui-même se porter préjudice, d'aucuns pourront-ils venir à bout de l'en trouver coupable ${ }^{61}$ ?»

Et, dans la seconde partie, il développe encore plus l'accusation portée contre le jeu :

« Tout jeu devient coupable s'il est ainsi constitué qu'il attise les passions d'un être humain, et particulièrement d'un jeune être chez qui, à cause de la vivacité, la mèche prend d'autant plus facilement feu et peut aisément s'enflammer, en particulier de dépit, de colère, d'avidité et de cupidité et de pareilles affections. Le jeu est et devient coupable si le plaisir qu'il donne dévoie un être et lui dérobe le temps dont il est redevable à d'autres occupations. Et s'il enchaîne l'homme de telle sorte que celui-ci mette dans le jeu d'argent ce dont il a besoin pour d'autres nécessités, si par là il se ruine ou dépend des autres de sorte qu'avec le jeu il s'assujettisse au vol confirmé, ou encore s'il se lèse soimême de grossière façon. L'expérience enseigne quelle peste est le jeu pour les jeunes gens et combien d'entre eux, sous l'influence de cette fièvre maudite, sont précipités sur de fausses routes et même dans l'abîme de la temporelle corruption. L'expérience enseigne que souvent s'y mêle l'ambition et que celle-ci, non seulement désunit les meilleurs amis, les frères, les époux, mais encore qu'elle envahit jusqu'à l'âme $[M u t h]$ où elle fait naître le meurtre et l'assassinat. Les grands jeux de hasard sont des sources tout particulièrement fécondes de toutes ces conséquences dommageables et épouvantables, ce pourquoi sans doute la sagesse des gouvernants réclame l'interdiction de ces jeux qu'accompagnent l'injustice, la séduction, le vol ainsi que de pareilles conséquences épouvantables, ainsi que la protection de l'État contre de tels tyrans qui jettent tant de notre légère jeunesse dans un esclavage infortuné, l'aveuglent et la peuvent porter jusqu'à la folie furieuse. Mais de tels jeux devraient, non seulement dans des maisons privées, mais encore dans toutes les réunions, assemblées, bals masqués publics, à la cour et partout, être interdits

61. Ibid., p. 395-396. 
de la manière la plus rigoureuse; les châtiments les plus durs, non seulement la confiscation de l'argent du jeu, mais encore l'emprisonnement, la dégradation et même l'infamie, devraient nécessairement y être attachés, et il faudrait que rien ne fût laissé en dehors de la rigueur de la loi et des châtiments, jamais et en aucune circonstance. C'est par une telle rigueur que Louis XIV a banni le crime hors de France et a entièrement réprimé les duels autrefois si répandus dans cette nation. Les grands jeux de hasard et d'argent sont tout autant des ennemis de l'État et méritent la même rigueur; et il serait sage que toutes les maisons publiques de jeux fussent complètement supprimées, parce que, dans une République, elles sont le chemin qui mène à la corruption des mœurs de la jeunesse, qui, par là, peu à peu, est prise dans les rets et enchaînée par les passions. On commence par les petites choses, on finit par les grosses, et l'on y vient peu à peu, et ainsi de suite. Toutes ces choses sont choses d'expérience. La philosophie et la politique [Philosophie und Politic] s'accordent ici parfaitement avec la religion chrétienne [Christenthum]. Celui qui aime ses concitoyens et ses sujets ne peut point autrement penser ${ }^{62}$. "

On voit enfin explicitées les motivations profondes de cette réflexion sur les rentes viagères : la philosophie ${ }^{63}$, la politique et la religion chrétienne $y$ sont parfaitement accordées, tout comme la Révélation, la raison et l'expérience, cette autre Sainte Trinité. Obéir en tout à l'ordre de Dieu, c'est s'assurer d'un bonheur terrestre. On notera que, dans ce domaine, les considérations populationnistes sont absentes.

De plus, et pour une bien rare fois, un point de la politique de Louis XIV est approuvé, certes entièrement dans l'ordre de la morale publique : la condamnation des jeux est comparée à l'interdiction des duels. Dans la représentation que se fait le pasteur, les maisons de jeux posent bien une question de vie et de mort. Le tripot est la perdition. La question morale est mise ici au tout premier plan : l'État frédéricien, le despotisme éclairé, sera moral ou ne sera pas, à ceci près que Süßmilch parle en théologien tandis que Frédéric II agira en souverain sceptique et incroyant.

Süßmilch rappelle alors brièvement l'objet du débat : les rentes, elles, sont lavées de tout péché :

« Mais, si l'on veut, dans un sens étendu, nommer jeu ce commerce des rentes, je ne crois pas que l'on y puisse montrer la moindre chose qui puisse rendre un jeu coupable, ce pourquoi il est, par conséquent, préservé de toute accusation $^{64}$, »

Pourtant, les rentes ne sont pas exemptes de tout risque et Süßmilch le reconnaît lui-même :

62. Ibid., p. 396-397.

63. Terme que Süßmilch emploie fort rarement.

64. SÜssmiLCH, 1761-1762, vol. II, chap. XXIII, § 514, p. 397. 
«Le savant Monsieur le professeur Hanow de Dantzig a, il y a quelque temps, expressément écrit une profonde dissertation sur la moralité des tontines et des rentes viagères [...] Il y est montré comment un État souffre par trop des préjudices d'intérêts élevés et comment l'acheteur d'une rente peut faire son malheur s'il prétend hasarder toute sa fortune de manière irréfléchie. Au reste, ce commerce est sauvé de toutes les accusations infondées et est apprécié, non seulement comme équitable et utile, mais encore comme nécessaire à tous les grands besoins de l'État ${ }^{65}$. »

Il s'agit bien pour Süßmilch d'une morale du Mittel, de la moyenne. On peut faire son propre malheur si l'on hasarde sa fortune de manière irréfléchie, mais le rentier est un joueur raisonnable. Un joueur rationnel?

\subsection{La question de l'emprunt forcé : État frédéricien et Providence}

Après ces envolées, Süßmilch se demande si l'État, en cas d'urgence, guerre ou grands travaux d'amélioration, a le droit de contraindre les sujets à acheter des rentes viagères, autrement dit s'il est possible de justifier l'emprunt forcé.

La réponse se fait en deux temps pour le cas de la guerre : il ne fait aucun doute que le souverain, pour la défense de la patrie, n'a pas besoin de recourir à l'emprunt forcé. Il est en droit, selon Süßmilch, de recourir à l'imposition extraordinaire, c'est-à-dire de n'être pas astreint au remboursement. Le sujet ne prête pas, il donne. L'argument dévoile le patriotisme de Süßmilch ${ }^{66}$ : puisque le devoir [Pflicht] est de donner son sang, il ne faut pas renâcler, a fortiori, à donner son argent :

« Posons le cas d'une guerre dans laquelle le prince a besoin d'argent pour la défense de la patrie. Ici, il ne fait aucun doute qu'il faut répondre oui à cette question. Un prince est en pareil cas justifié d'exiger de ses sujets des contributions extraordinaires, par la capitation [durch Kopfsteuer] ou d'une autre manière, sans qu'il lui soit nécessaire de leur promettre le remboursement. Ce droit de l'État se fonde sur la conservation de son bien propre et de la patrie, à quoi un pays est assujetti par le devoir. Et si le sujet est obligé de protéger la patrie par le sacrifice de sa propre vie et de porter au besoin les armes, combien plus encore est-il obligé de lui venir en aide avec son bien, le moindre étant son sang, sans réclamer de rémunération en retour? Le devoir de la société civile peut réclamer de nous notre sang et notre bien, la constitution de l'État fût-elle aussi libre et républicaine [republicanisch] que possible. Rien ne nous peut acquitter de cela ${ }^{67}$. 》

65. Ibid., p. 397-398.

66. C'est une autre question de savoir si ce patriotisme était de commande : on sait que Süßmilch a écrit de vibrants sermons d'actions de grâces après les victoires de Frédéric et qu'il n'a pas cessé de vilipender la guerre et les guerres de conquêtes de Louis XIV...

67. Süssmilch, 1761-1762, vol. II, chap. xxiII, $\$ 515$, p. 398-399. 
Il ne s'agit pas là de commerce, mais de devoir et de conscience. Ensuite, tout dépend de l'attitude du souverain. Et nous assistons, nous semble-t-il, à un panégyrique de l'État frédéricien. La question n'est plus celle de l'impôt extraordinaire mais bien celle de l'emprunt forcé. Mais «forcé » ne peut plus du tout alors avoir le sens de « contraint ». Ce n'est que par sa bonne volonté, "s'il a en tête d'être paternel envers ses enfants " [väterlich gegen seine Kinder] que le souverain peut, au lieu de recourir à l'impôt extraordinaire, utiliser l'emprunt forcé, lequel apparaît donc plutôt comme une grâce souveraine que comme un scandale. Dans l'État frédéricien, tout le monde est commis [Bedienter], "officier», employé de l'État, y compris, soi-disant, le prince qui en est le premier serviteur. Alors le sujet, s'il n'est pas mort au combat - mais Süßmilch n'envisage pas cette hypothèse - , récupérera son bien avec intérêts :

"Mais si un souverain conduisant la guerre a en vue d'être paternel à l'égard de ses enfants [seine Kinder] et se propose de leur redonner la partie du bien qu'ils lui ont donnée, non seulement en elle-même, mais encore avec les fruits et les intérêts qu'elle aurait pu acquérir dans l'intervalle, peut-on alors douter qu'il ne soit juste qu'il exhorte les âmes ingrates et récalcitrantes à contribuer par les moyens appropriés? Cette manière de rapporter bientôt, par les intérêts, un important capital a, en vérité, en elle-même tant de séduction qu'aucune contrainte n'y semble nécessaire ${ }^{68}$."

Et nous voici revenu, insensiblement, aux emprunts de l'État par rentes interposées. La morale de l'histoire est que l'État a toujours raison en la matière :

"L'expérience enseigne assez que beaucoup d'Allemands achètent des rentes dans des sociétés étrangères, en Hollande, en Angleterre et en France. Mais, comme l'ignorance, ou encore l'esprit de dénigrement et de méchanceté, peut noircir les meilleurs desseins de l'Etat par des préjugés forgés et des appréhensions sans fondement, et empêcher ou retenir la bonne volonté à contribuer à de tels emprunts [Anlehn], de tels moyens de coercition sont, non seulement permis, mais encore nécessaires ${ }^{69}$."

La chose est donc claire.

Suit une courte dissertation qui montre à la fois l'avantage, pour le pauvre, de la rente par rapport à l'impôt, et le moyen de proportionner ce dernier pour ne léser personne et ne pas commettre d'injustice. L'ordre de la mortalité conduit à l'ordre moral et à l'ordre politique qui en découle :

68. Ibid., p. 399 .

69. Ibid., p. 399-400. 
«Dans combien, oui dans combien d'innombrables autres cas ne faut-il pas que le bien commun soit conservé par la rigueur de la loi? [...] L'expérience nous a de tout temps enseigné qu'il n'était pas plus difficile d'inventer de certains principes [Principia] par quoi proportionner les contributions de sorte qu'il ne soit fait tort à personne et que la justice n'en soit pas lésée. Par un tel emprunt, le pauvre demeure tout à fait préservé, l'homme de condition moyenne et les riches supportant à eux-seuls le tribut. La partie plus raisonnable donne librement et avec joie, à cause de l'espérance de gain; la plus obtuse [der finstere], toujours mécontente, la partie peureuse et avare est, elle, contrainte de le faire et ne peut se séparer de la patrie, et elle n'a également aucun motif de plainte puisqu'elle récupère le capital et les intérêts et $a$, dans une tontine, l'espérance de le faire de manière multipliée ${ }^{70}$. "

Rêve financier ou utopie actuarielle du remplacement de l'imposition par des emprunts d'État sous forme de rentes? Süßmilch — qui n'a pu l'être dans la réalité politique - se laisse ici aller à la griserie du conseiller de cabinet. Enfin, le bien commun doit être conservé par la rigueur de la loi : fort belle maxime pour le despotisme éclairé. Mais le roi ne choisira ni les tontines ni les rentes. Süßmilch semble avoir oublié que Frédéric II ne partage pas sa foi qui repose sur l'ordre de la mortalité, ordre même de Dieu, essentiel et intangible, partie intégrante de la Création, puisque, comme la terre, cet ordre est créé pour l'homme et sa souveraineté.

Comment résumer cette philosophie politique? Il s'agit incontestablement de promouvoir l'ordre et l'autorité, de même que l'esprit d'entreprise. Süßmilch se situe au moment où le caméralisme mercantiliste va céder le pas au précapitalisme industriel. Son analyse de 1761-1762 n'est en tout cas plus celle d'un humaniste désireux de servir exclusivement son prochain. Ce prochain est devenu l'État frédéricien et les objectifs sont à présent clairement économiques. Le problème de l'assurance s'est estompé pour céder la place à celui de l'impôt, de l'emprunt et de l'enrichissement de l'État.

Ce n'est pas au nom d'une future « démographie » que Süßmilch entreprend, après ses prédécesseurs, d'explorer le domaine de la mortalité, mais dans un dessein à la fois utilitaire et apologétique. Utilitaire, parce que le calcul des rentes viagères et de ce qui ne s'appelle pas encore les assurances y est subordonné. Apologétique, parce que c'est par l'ordre de la mortalité que se manifeste le plus évidemment la providence de Dieu. La mort équilibre certes la naissance, mais apparaît également comme une énigme dans le dessein divin, notamment si elle résulte de catastrophes, de

70. Ibid., p. 400. 
maladies, ou s'il s'agit de la mort d'un enfant ou d'une jeune personne qui n'aurait pas encore, en apparence, accompli son temps. La mort est étroitement liée à la question du mal. Déclarer qu'un ordre y règne également, c'est considérer que les choses ne sont pas livrées à une fortune aveugle et insensée.

C'est également faire de l'ordonnance du plan général de L'Ordre divin un reflet, esquisse imparfaite mais vraisemblable [wahrscheinlich], du dessein général de Dieu. Pour approcher ce dessein, nous voyons Süßmilch utiliser les rudiments du jeune calcul des probabilités, afin de parvenir à cette "vraie semblance " qui n'est pas tout à fait le vrai, mais qui s'en approche assez pour que la prudentia humaine, qui doit être conforme à la providentia divine, s'y applique sans trop errer : principe même de cette physico-théologie qui prend ici tout son poids d'élément déterminant dans la naissance des sciences sociales.

Jean-Marc RoHRBASSER (septembre 1997). 


\section{BIBLIOGRAPHIE}

[ANONYME], 1787, Traité d'arithmétique à l'usage des pensionnaires et des écoliers des frères des Écoles chrétiennes, Rouen, chez veuve Laurent Dumesnil.

BéHAR (Cem L.), 1976, « Des tables de mortalité aux XVII et XVIII ${ }^{e}$ siècles. Histoire, signification ", Annales de démographie historique, p. 173-200.

Bonar (James), 1931, Theories of population from Raleigh to Arthur Young. Lectures delivered in the Galtonian Laboratory, University of London, under the Newmarch Foundation, Febr. 11 to March 18, 1929, with two additional lectures and with references to authorities, Londres, George Allen \& Unwin.

DE WITT (Jan), 1671, Waerdije van Lijf-Renten Naer proportie van Los-Renten [La valeur des rentes viagères en proportion des rentes amortissables], Amsterdam.

Deparcieux (Antoine), 1746, Essai sur les probabilités de la durée de la vie humaine [...] par M. Deparcieux, de la Société royale des sciences de Montpellier. Objections faites à $M$. Deparcieux, des Académies royales des sciences de Paris et de Berlin, sur son Livre des Probabilités de la durée de la vie humaine : avec les réponses à ces objections, Paris, chez les frères Guérin.

DUPÂQUIER (Jacques), 1985, " Providence et probabilité », Revue de synthèse, 117, janv.-mars, p. 81-99.

DUPÂQUIER (Jacques et Michel), 1985a, Histoire de la démographie. La statistique de la population des origines à 1914, Paris, Librairie académique Perrin (« Pour l'histoire »).

DUPÂQuiER (J.), 1985b, «Le Mémoire de Jean De Witt sur la valeur des rentes viagères », prés. et comment. par J. DupÂQuier, trad. P. J. L. DE ChateleuX, Annales de démographie historique, p. 355-394.

DUPÂQUIER (J.), 1996, L'Invention de la table de mortalité. De Graunt à Wargentin, 1662-1766, Paris, Presses universitaires de France.

GraunT (John), 1676, Natural and Political Observations mentioned in a following index and made upon the Bills of Mortality, by capt. John Graunt, fellow of the Royal Society. With reference to the government, religion, trade, growth, air, diseases, and the several changes of the said city [...] The fifth edition much enlarged, Londres, printed by John Martyn [Observations naturelles et politiques répertoriées dans l'index ci-après et faites sur les Bulletins de mortalité par John Graunt citoyen de Londres en rapport avec le gouvernement, la religion, le commerce, l'accroissement, l'atmosphère, les maladies et les divers changements de ladite cité, éd. critique et trad. Éric VILQUIN, Paris, Institut national d'études démographiques, 1977].

HALD (Anders), 1990, A history of probability and statistics and their applications before 1750, New York, John Wiley and sons («A Wiley-Intersciences publication $")$.

HALLEY (Edmund), 1693a, " An estimate of the degrees of the mortality of Mankind, drawn from curious Tables of the Births and Funeral's at the city of Breslaw; with an attempt to ascertain the price of annuities upon lives, by M. E. Halley F.R.S. », Philosophical Transactions, January, 196, p. 596-610. 
HaLley (E.), 1693b, «Some further considerations on the Breslaw Bills of Mortality. By the same hand, \& c. », Philosophical Transactions, January, 196, p. 654-656, in Mortality in pre-industrial times. The contemporary verdict, with an introd. by James H. CASSEDY, Westmead, Gregg International Publ., 1973.

Huygens (Christiaan), 1669, Euvres complètes de Christiaan Huygens, publ. par la Société hollandaise des sciences. T. VI : Correspondance 1666-1669, La Haye, Martinus Nijhoff, 1895.

Leibniz (Gottfried Wilhelm), 1680a, "De Aestimatione Redituum ad Vitam », in L'Estime des apparences, 21 manuscrits de Leibniz sur les probabilités, la théorie des jeux, l'espérance de vie, textes établ., trad. et annot. par Marc PARMENTIER, Paris, Vrin (« Mathesis »), 1995, p. 292-313.

Leibniz (G. W.), 1680b, «Lebenserwartung I », in G. W. Leibniz, Sämtliche Schriften und Briefe, hrsg. vom Zentralinstitut für Philosophie an der Akademie der Wissenschaften der DDR. Vierte Reihe : Politische Schriften; dritter Band: 1677-1689, Berlin, Akademie-Verlag, 1986, p. 449-452.

Leibniz (G.W.), 1680c, « Lebenserwartung II », in G.W. LeibNIz, Sämtliche Schriften und Briefe, hrsg. vom Zentralinstitut für Philosophie an der Akademie der Wissenschaften der DDR. Vierte Reihe : Politische Schriften; dritter Band: 1677-1689, Berlin, Akademie-Verlag, 1986, p. 452-456.

LEIBNIZ (G.W.), 1680d, «Essay de quelques raisonnemens nouveaux sur la vie humaine et sur le nombre des hommes », in G.W. LeIBNIz, Sämtliche Schriften und Briefe, hrsg. vom Zentralinstitut für Philosophie an der Akademie der Wissenschaften der DDR. Vierte Reihe : Politische Schriften; dritter Band : 16771689, Berlin, Akademie-Verlag, 1986, p. 456-467.

LiTtré (Émile), 1877, Dictionnaire de la langue française, Paris, rééd. Encyclopædia Universalis brit., 1983, 7 vol.

PEarson (Karl), 1977, The History of statistics in the seventeenth and eighteenth centuries. Conférences prononcées à Londres en 1921-1933, éd. par Egon S. Pearson, Londres, Ch. Griffin and Co.

PetTy (sir William), 1670-1676, Political Arithmetick or a discourse concerning the extent and value of lands, people, buildings, husbandry, manufacture, commerce, fishery, artizans, seamen, soldiers, publick revenues, interest, taxes, valuation of men, increasing of seamen, of militia's herbours, power at sea \&c. as the same relates to every country in general but more particular to Great Britain \& c., in Euvres économiques de sir William Petty, trad. de l'anglais par Henry Dussauze et Maurice Pasquier, Paris, chez Giard et Brière, 1905.

ROHRBASSER (Jean-Marc), VÉron (Jacques), 1998, « Leibniz et la mortalité : mesure des "apparences" et calcul de la vie moyenne », article à paraître dans Population, 1 .

STRUYCK (Nicolas), 1740, Inleiding tot de algemeene Geographie [Introduction à la Géographie générale]. [Avec] Gissingen over de Staat van het menschelyk Geslagt en de Uitreekening der Lyfrenten [Hypothèses sur l'État de l'espèce humaine suivies du calcul des rentes viagères], Amsterdam, Isaak Tirion.

STRUYCK (N.), 1753, Vervolg van de beschrijving der staartsterren, en nader ontdekkingen omtrent den staat van het menchelijk geslagt, uit ondervindingen opgemaakt, benevens eenige stermerkingen [Suite de la description des comètes et découvertes plus détaillées concernant l'état du genre humain, basées sur des expériences, avec quelques observations astronomiques], Amsterdam, Isaak Tirion.

STRUYCK (N.), 1912, Les Euvres de Nicolas Struyck (1687-1769) qui se rapportent au calcul des chances, à la statistique générale, à la statistique des décès et 
aux rentes viagères, tirées des Euvres complètes, et traduites du hollandais par J.A. Vollgraff, offertes aux membres du Septième Congrès international des actuaires, réunis à Amsterdam en septembre 1912 par la Société générale néérlandaise d'assurances sur la vie et de rentes viagères, Amsterdam.

SüssmILCH (Johann Peter), 1741, Die göttliche Ordnung in den Veränderungen des menschlischen Geschlechts, aus der Geburt, Tod, und Fortpflanzung desselben erwiesen von Johann Peter Süßmilch, Prediger beym hochlöblichen Kalcksteinischen Regiment. Nebst einer Vorrede Herrn Christian Wolffens [L'Ordre divin dans les changements de l'espèce humaine, démontré par la naissance, la mort et la propagation de celle-ci par J.P. Süßmilch, prédicateur au très estimable régiment Kalckstein. Avec une préface de Monsieur C. Wolff], Berlin, zu finden bey J.C. Spener.

Süssmilch (J.P.), 1761-1762, Die göttliche Ordnung in den Veränderungen des menschlichen Geschlechts, aus der Geburt, dem Tode und der Fortpflanzung desselben erwiesen von Johann Peter Süßmilch, Königl. Preuß. Oberconsistorialrath, Probst in Cölln, und Mitglied der Königl. Academie der Wissenschaften [L'Ordre divin dans les changements de l'espèce humaine, démontré par la naissance, la mort et la propagation de celle-ci, par J.P. Süßmilch, conseiller du Consistoire supérieur royal de Prusse, prévôt à Cölln et membre de l'Académie royale des sciences], Zwote und ganz umgearbeitete Ausgabe, Berlin, Im Verlag des Buchladens der Realschule, 2 vol.

SüssMilch (J.P.), 1776, Die göttliche Ordnung in den Veränderungen des menschlichen Geschlechts, aus der Geburt, dem Tode und der Fortpflanzung desselben erwiesen von Johann Peter Süßmilch, gewesenen Königl. Preuß. Oberconsistorialrath, Probst in Cölln, und Mitglied der Königl. Academie der Wissenschaften, Berlin, Im Verlag der Buchhandlung der Realschule, 3 vol. Dritter Theil, welcher Anmerkungen und Zusätze zu den beyden ersten Theilen nebst einer Abhandlung von Wittwenverpflegungsgesellschaften enthält, hrsg. von Christian Jacob Baumann, Prediger zu Lebus [Avec une troisième partie qui contient des observations et des additions aux deux premières parties, et un traité sur les sociétés d'assistance aux veuves, publ. par C. J. Baumann, prédicateur à Lebus].

THIVEAud (Jean-Marie) et al., 1989-1990, Finances et démocratie ou Le peupleprovidence. Mélanges sur le crédit, l'épargne, la prévoyance et la dette publique, 1750-1850. Recueil d'articles de Jean-Marie Thiveaud, Fabrice Laroulandie, Alex Doppia et Zheng Kang, Paris, Mission des travaux historiques de la Caisse des dépôts et consignations. 